\title{
Gravitationally frozen objects and relativistic explosion in general relativity: frozars, frozons and superbursts
}

\author{
Zahid Zakir $^{1}$
}

\begin{abstract}
In general relativity (GR) the worldline of a particle is unique and invariant, proper time and world time $t$ are two parametrization of the same events on it only, that leads to a constraint for the proper time moments relating them by $t$. So, at contraction of a dust shell the proper times at finite $t$ asymptotically freeze by no reaching a moment when the surface could cross the gravitational radius. Processes in entire volume of a star freeze at first at the center, then at higher layers, and at last the surface freezes outside the gravitational radius. Therefore in GR contraction leads to formation not black holes, but frozars (from "frozen star") with the gravitationally frozen state of matter in entire volume, where the worldlines of particles are time-like everywhere, parallel to the $t$-axis and each other. Frozar formation in GR is shown for a thin dust shell, a dust ball, a star of uniform density and stars with ultrarelativistic matter. In real stars local temperatures in layers grow faster than temperature on the surface, and the last one grows on $t$ exponentially fast. As high star's mass, as high probability of that freezing occurs faster than warming up and the frozar will has time to be formed. But at lower masses, when the freezing does not enough fast, the warming up can stop contraction and can lead to explosion. During contraction a significant part of matter appear near the surface where in GR the physical volume sufficiently grows and energy of contraction is transformed to heat with transition of matter to the radiation dominated phase. If the star did not has time to be frozen, the part of ultrarelativistic matter and radiation leaves the star quickly, which appears as relativistic explosion, and the object will observed as relativistic supernova or hypernova. The observed lack of frozars of 2-4 solar mass and flat character of mass spectrum of more massive candidates to frozars confirm these predictions of the theory. The Big Bang and some explosions in astrophysics with large energy release probably are the cases of the relativistic explosion. In the frozar theory it appears a new GR phenomenon, the gravitational crystallization, defining structure of the most compact and massive objects in particle physics, astrophysics and cosmology. Gravitational radius of the system of few frozars sufficiently exceeds the radius of each of them and, therefore, at closing up they will not be able to merge and become frozen at distances larger of their radii, forming a new state of matter - the gravitational crystal. Frozons, particles of the Planck energy, quantum fluctuations of which are frozen in their self gravitational field, also can not merge, i.e. for frozons there will be no interaction vertexes and they form only clusters and gravitational microcrystals. In astrophysics the supermassive frozen objects in the centers of star clusters, galaxies and quasars are probable gravitational supercrystals from frozars and ordinary matter. Relic frozons and frozar crystals could be the centers of inhomogeneities and also could be appear as a dark matter. If there is the backward contraction, the Universe as whole can be frozen also in the state of a global gravitational crystal which would stop the contraction.
\end{abstract}

PACS: 04.20.Dg; 04.70.-s; 97.60.-s, 98.54.-h

Keywords: relativistic stars, gravitational collapse, black holes, quark stars

${ }^{1}$ Centre for Theoretical Physics and Astrophysics, Tashkent, Uzbekistan, zahidzakir@ theor-phys.org 


\section{Content}

Introduction .42

1. Frozar formation at cold contraction of stars

1.1. Freezing of a test particle in the field of contracting star ...........................................44

1.2. Freezing of a dust shell with formation of a simplest frozar ....................................47

1.3. Freezing of a dust ball with transition to the frozar state ...........................................49

1.4. Freezing of a star with nonzero pressure with transition to frozar ...............................53

2. Relativistic heating at contraction of stars and relativistic explosion ...........................55

2.1. Heating at contraction and relativistic explosion of supermassive stars ......................55

2.2. Heating at contraction and relativistic explosion of superdense stars ..........................56

2.3. Big Bang as relativistic explosion of the Universe ..................................................57

3. Gravitational crystallization at different scales........................................................58

3.1. Impossibility of merging of frozars and gravitational crystallization .........................58

3.2. Gravitational crystallization at the Planck distances .................................................59

3.3. Cosmological consequences of gravitational crystallization.......................................61

4. Astrophysics of frozars and relativistic explosion .................................................62

4.1. The expected and observed properties of stellar mass frozars .................................62

4.2. Supermassive frozars in galaxy nuclei as gravitational crystals .................................64

4.3. The expected and observed properties of relativistic explosions .................................65

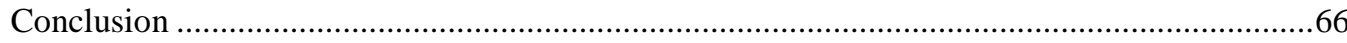

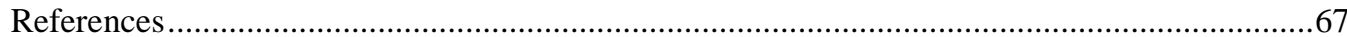

\section{Introduction}

The destiny of massive stars at their gravitational contraction has remained long time as one of main unsolved problems of relativistic astrophysics [1-8]. In the Newtonian theory's scenario the matter of a star of mass $M>3 M_{\odot}$ collapses inside the gravitational radius $r_{g}=2 G M / c^{2}$, playing the role of the visibility horizon, and quickly falls to the center by forming the singularity. The question was what part of this scenario holds in general relativity (GR) and what part changes.

The mathematical aspects of the problem was solved already at initial stage for simple models of GR, including a dust shell and dust ball [1-4]. The next stage was the distinction of a physical part of these solutions, which would allow to define scenarios of contraction and structure of compact relativistic objects. It was known that while the star's surface lies beyond $r_{g}$, in the star's internal metrics there are neither singularity, nor the horizon, and the descriptions of worldlines in terms of proper time $r(\tau)$ or world time $r(t)$ are different parametrizations of the same events only, i.e. $r(\tau) \equiv r(t)$.

The aroused problem was how to be with distinction of scenarios of contraction in terms of $\tau$ and $t$ in that part of the Newtonian evolution, when the surface (of radius $\left.r_{b}(t)\right)$ crosses $r_{g}$. In GR the trajectory in the region $r_{b}(t) \leq r_{g}$ in terms of $\tau$ formally is the same as in the Newtonian case, whereas at $t$-evolution this part is simply absent in the entire interval $t<\infty$. 
If the description of worldlines in terms of $\tau$ could be full, the Newtonian scenario with collapse inside $r_{g}$ could be realized, and the description in terms of $t$ could be incomplete. If the description in terms of $t$ occurs full, then the surface will never crosses $r_{g}$, i.e. $r(t)>r_{g}$ at $t<\infty$, and $\tau(t)$ will be frozen asymptotically $\tau(t)<\tau_{g}$ about the value $\tau_{g}=\tau(\infty)$, so the variability area of $\tau$ will not infinite $[0, \infty)$ as in the Newtonian theory, but it occurs finite $\left[0, \tau_{g}\right)$.

Thus, in GR the destiny of contracting stars depends on an answer to the question what of two parametrizations, $\tau$ or $t$, fully describes the worldlines of star's particles.

The Black Hole Hypothesis (BHH) [4-8], which has been considered as a paradigm, supposes that the Newtonian scenario of collapse with $\tau$-description is full and the $t$-description is incomplete. The criticism of BHH is presented in [10].

The Frozar Theory (FT, see $[9,10]$ ) is based on the fact that in GR $t$-evolution completely describes each event along the worldlines of particles of the star during the existence time of the real Universe $(t<\infty)$. Then instead of the collapse inside $r_{g}$ there is a gravitational freezing of matter by transition of a dust star to the state of a gravitationally frozen star, or frozar. Thus, the evolution of a particle on the star's surface at $t<\infty$, both in terms of $t$ and $\tau(t)$, is described only by a unique set of real events on the invariant worldline of the particle. As a result, the surface remains outside $r_{g}$ for all observers during all the existence time of the universe, since the transition from $t$ to $\tau(t)$ does not change the freezing of $\tau$ with respect to $t$. For this reason the gravitational contraction in GR and structure of objects are described adequately only by FT.

A physical basis for such treatment is a new phenomenon which is absent in the Newtonian theory and which stabilizes contraction in GR by excluding the collapse inside $r_{g}$. It is the gravitational delay of proper times $(\tau)$ with respect to $t$ - the phenomenon which was predicted by GR and observed, and now it is using even in engineering practice (in satellite positioning systems) [11], but still was not adequately understood and taken into account in particle physics and astrophysics. In the field of the contracted dust star it leads to the asymptotic freezing of all processes in entire volume of the star, so that internal layers freeze before the surface and $r_{b}(\tau) \equiv r_{b}(t)>r_{g}$ at $t<\infty$ for any observers with any coordinates.

For the real stars the accounting of pressure and temperature can lead to quasistabilization of contraction with formation of a glowsar ("glow star"). But it is more probable the bounce with the relativistic explosion when a part of the rest energy of matter, hot on the local temperature, is radiated outside in the form of a superburst.

The frozar theory also predicts the formation of a new state of matter - massive and supermassive frozar clusters containing two or more frozars and ordinary matter. Falling of ordinary matter to the existing frozar leads only to increasing of mass of the latter, whereas when two or more frozars fall to the common center of inertia there appears a new situation. In the Newtonian physics the compact objects can merge by 
forming a new more massive compact object. In GR at falling of two and more frozars (or glowsars) they can not merge due to freezing entirely on the gravitational radius of the system by forming a frozar cluster. For example, in case of three equal mass frozars the gravitational radius of the system is three times larger than the radius of each of frozars.

Particles of the the Planck energy also pass into the state of a frozon - a particle the fluctuations which are frozen in its self gravitational field $[9,10]$. Two and more frozons, at closing, also cannot merge and form the frozon clusters only.

In Part 1 of the paper the formation of a frozar at contraction of a dust matter is considered, in Part 2 the contraction of stars at nonzero pressure and temperature with formation of glowsar and also the possibility of relativistic explosion are discussed. In Part 3 the hierarchy of frozon and frozar clusters in particle physics, astrophysics and cosmology is considered. In Part 4 the astrophysical consequences of the frozar theory and its confrontation with observations are discussed.

\section{Frozar formation at cold contraction of stars}

\subsection{Freezing of a test particle in the field of contracting star}

Let's consider a test particle free falling in the gravitational field of a spherical star. Let star's surface free falls too, but had began to fall before the test particle, so at any time the particle moves beyond the star in its static field.

For such extended object as a spherical nonrotating star a rest frame $K_{0}$ is that where its center of inertia is rested, i.e. the worldlines of its center and a distant observer, also rested in $K_{0}$, are parallel to the $t$ axis of $K_{0}$ near the distant observer. For the distant observer the $K_{0}$ is inertial frame where it can be defined a conserving "rest energy" $E_{0}=M c^{2}$ of the star as whole and only in $K_{0}$ the gravitational field around the star is static and spherically-symmetric.

In GR the simultaneity of events in a static field is defined globally and initial synchronization of all clocks is provided as in flat spacetime. However, gravitation slows down proper times $\tau$ and, therefore, near the star the initial synchronization of rested in $K_{0}$ standard clocks will be broken at subsequent moments. But, slowing down of local processes does not break the global simultaneity of events in this static field. A new with respect to flat spacetime will only that for standard clocks at different radial distances there will simultaneous their different indications. Therefore, for the expression of simultaneity of events at different distances the coordinate clocks are necessary in $K_{0}$ which everywhere and at any moment go synchronously, by showing world time $t$ on hypersurfaces of simultaneity $t=$ const . For this purpose the coordinate clocks should go as faster as the standard clocks of $K_{0}$ at any point are slowed down by gravitation.

Thus, in the $K_{0}$ the events on the surface and outside the star are marked both by $\tau$, showing the local rate of physical processes, and by $t$, showing what of events on the surface and outside the star occur simultaneous with the moment $t$ of the distant observer's proper time. World time $t$ is also the "proper time of the star as whole" without internal motion of its particles and gravitation time delay. Therefore, in $K_{0}$ true physical properties of the star are expressed without kinematical complications and distortions. 
Notice, that the attempts to describe the whole star's evolution in terms of a set of moving local frames, comoving to the falling particles, do not enter a new information than the description in $K_{0}$, but only distort this simple picture. Particularly, for each falling observer in them the star's field is non-static and non-spherical. Moreover, each of radially falling local systems has a proper hypersurface of simultaneity and when extended frames of reference are constructed by joining of such local frames, here it appears the problem with causality. Only in $K_{0}$, constructed from relatively rested local frames and where there is a single, common for all them, hypersurface of simultaneity $t=$ const., there are no problems with causality due to joining everywhere the simultaneous events only.

The frame $K_{0}$ with the curvature coordinates $(t, r, \theta, \varphi)$ is physically distinguished in the sense that only in it: 1) simultaneity of events is defined globally; 2) the intervals of $t$ in the field everywhere and always are equidistant; 3 ) radial velocities of particles on a thin layer are the same; 4) thin layers are spherically-symmetric. In any other extended frame of reference some of these four properties are absent, which complicates the description.

The spacetime metrics on and outside $\left(r \geq r_{b}\right)$ the spherical non-rotating (and neutral) star in the curvature coordinates is given by the Schwarzschild solution $(c=1)$ :

$$
d s^{2}=\left(1-r_{g} / r\right) d t^{2}-\left(1-r_{g} / r\right)^{-1} d r^{2}-r^{2}\left(d \theta^{2}+\sin ^{2} \theta d \varphi^{2}\right), \quad r_{g}=2 G M .
$$

At radial falling of a particle ( $d \theta=d \phi=0$ ) in this field from large initial radius $r\left(t_{0}\right) \gg r_{g}$ (at $t_{0}=0$ and $\tau_{0}=0$ ), after large enough time interval $t \gg r_{g}$, when $r$ becomes very close to $r_{g}$, its worldline $r=r(\tau) \equiv r(t)$ in terms of two times $\tau$ and $t$ is expressed by the relations:

$$
\begin{gathered}
r(\tau)=r_{0} \cdot\left(1-\tau / \alpha_{0}\right)^{2 / 3}, \\
r(t) \simeq r_{g}\left(1+4 e^{-t / r_{g}}\right),
\end{gathered}
$$

where $\alpha_{0} \equiv 2 r_{0}^{3 / 2} / 3 r_{g}^{1 / 2}$. Both formulas (2) and (3) describe the same particle in the same spatial point $(r, \theta, \varphi)$ in terms of indications of two types of clocks. Therefore, by excluding from them $r$ we obtain a relationship $\tau(t)$ between the moments of two times at $t \gg r_{g}[9,10]$ :

$$
\tau(t)=\tau_{g}-4 r_{g} e^{-t / r_{g}}<\tau_{g},
$$

where $\tau_{g}$ formally corresponds to the moment $t=\infty$ :

$$
\tau_{g} \equiv \tau(\infty)=\alpha_{0}\left(1-r_{g}^{3 / 2} / r_{0}^{3 / 2}\right) .
$$

At changing of parametrization along the particle's worldline the moments of world time in the infinite interval $t \in[0, \infty)$ one to one correspond to the moments of proper time in the finite interval $\tau(t) \in\left[0, \tau_{g}\right)$. This means that $\tau(t)<\tau_{g}$ at any $t<\infty$ and for the falling particle the moment $\tau_{g}$ will not come never (Fig. 1).

It is natural to represent the particle's worldline $r=r[\tau(t)] \equiv r(t)$ on the $(t, r)$ plane (Fig. 2), where unit intervals of the $t$ axis are equidistant, $t$ is defined globally and is 


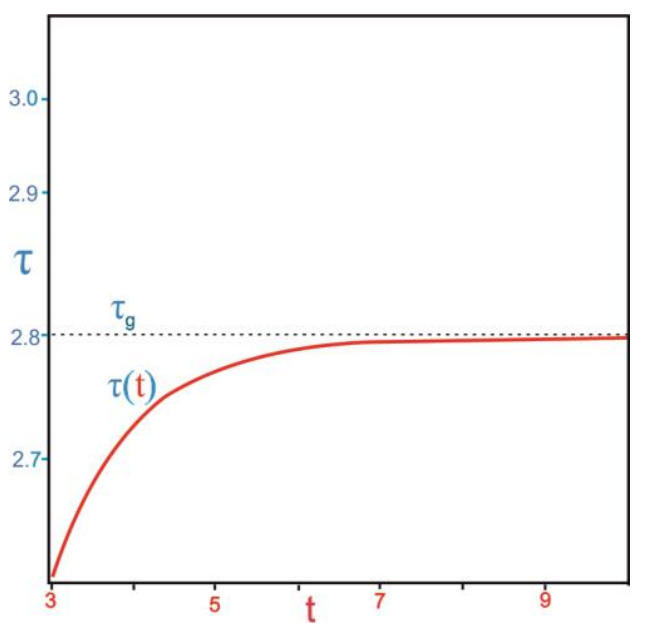

Fig. 1. At $t<\infty$ the proper time moments $\tau=\tau[r(t)]=\tau(t)$ of a falling test particle are constrained due to a relation by moments of $t: \quad \tau(t)=\tau_{g}-4 r_{g} e^{-t / r_{g}}<\tau_{g}$.

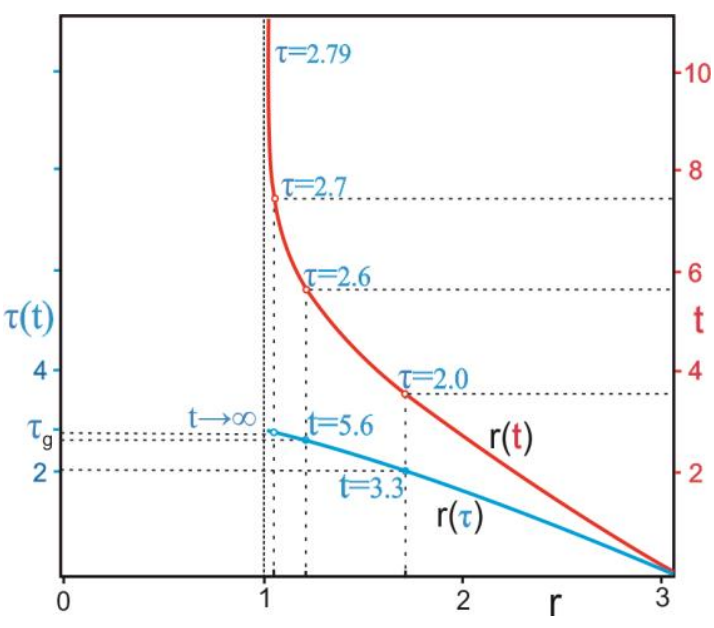

Fig 2. The worldline of a falling test particle $r(t)=r(\tau)$ in terms of $t$ and proper time $\tau$.

physically measurable. The proper times, however, are defined only locally and, therefore, the moments $\tau(r(t))$ can be defined only along the worldline, the unit intervals $\Delta \tau(t)$ are non-equidistant and stretching with respect to the units of $t$. At the uniform passage of $t$ the worldline asymptotically closes to $r_{g}$, the intervals $\Delta \tau(t)$ more and more stretch, and the values $\tau(t)$ become almost frozen, asymptotically approaching $\tau_{g}$ (Fig. 1).

The same worldline $r=r(\tau) \equiv r(t)$ on the $(\tau, r)$ plane (Fig. 2) looks so, as if the unit intervals $\Delta \tau$ are equidistant and the $\tau$ axis is defined globally, though $\Delta \tau$ are defined only locally and are different at different $r$. However, one can consider this axis conventionally as a one which observes a local falling observer. Then the moments $\tau(r)$ and $t(r)$ one can place along the worldline only, where the unit intervals $\Delta t$ will become non-equidistant and more and more contracted. At the uniform passage of $t(r)$ the worldline also asymptotically approaches $r_{g}$, and the freezing of the dust sphere visually appears as the "termination" of the worldline $r(\tau)$ near $r_{g}$. In fact the worldline is not broken, but asymptotically approaches $r_{g}$, and the evolution of $\tau(t)$ along it also goes in the form of asymptotical approaching $\tau(t) \rightarrow \tau_{g}$ at $t \rightarrow \infty$.

Thus, in GR the rate of $\tau(t)$ is strictly restricted by the rate of $t$ on hypersurfaces of simultaneity $t=$ const. and, therefore, at approaching $r_{g}$ by the particle the strong gravitation asymptotically freezes the particle's proper time. At $t<\infty$ its worldline is time-like $d s^{2}>0$ and the proper time interval $\Delta s^{2}=\Delta \tau^{2}=g_{\mu \nu} \Delta x^{\mu} \Delta x^{\nu}>0$ is invariant. Moreover, since in the static frame at a certain moment $t_{1}<\infty$ a proper distance between the surface and the gravitational radius is always positive defined $|\Delta r|>0$, then in any frame the invariant spacelike line element 
$\Delta s^{2}=-\Delta \mathrm{r}^{2}=g_{\mu \nu} \Delta x^{\mu} \Delta x^{v}<0$ will remains as spacelike. Observer on other frames will see the picture of freezing of $\tau$ with respect to $t$ with $r_{b}(\tau) \equiv r_{b}(t)>r_{g}$, even if they will express these two times through proper coordinates.

Particularly, if to enter Kruskal's coordinates $u$ and $v$, related by $r$ and $t$ as:

$$
\begin{aligned}
& u=\left(r / r_{g}-1\right)^{1 / 2} e^{r / 2 r_{g}}\left(e^{t / 2 r_{g}}+e^{-t / 2 r_{g}}\right) / 2, \\
& v=\left(r / r_{g}-1\right)^{1 / 2} e^{r / 2 r_{g}}\left(e^{t / 2 r_{g}}-e^{-t / 2 r_{g}}\right) / 2,
\end{aligned}
$$

then the spacetime interval (1) takes the form:

$$
d s^{2}=\frac{4 r_{g}^{3}}{r} e^{-r / r_{g}}\left(-d v^{2}+d u^{2}\right)-r^{2}\left(d \theta^{2}+\sin ^{2} \theta d \varphi^{2}\right) .
$$

Since at $t<\infty$ it is only a new parametrization of the same particle's worldline $r(t) \equiv r(u, v)$, then here to the inequality $r(t)>r_{g}$ corresponds the inequality $u(r, t)>v(r, t)$ for the new parameters. The crossing $r_{g}$ by the particle, i.e. the event $r(\infty)=r_{g}$, would take place only at the condition $u(r, \infty)=v(r, \infty)$, which formally could be occurred on this worldline only simultaneous with the event $t=\infty$, i.e. in the real world will never occur. Since the unique and invariant worldline of the falling particle at $t<\infty$ always lies outside $r_{g}$ of earlier contracted star, then any reparametrization of the same worldline can not to move the worldline of the particle on the surface inside $r_{g}$ and one will have again $r(t) \equiv r(u, v)>r_{g}$, but now only in the form of $u(r, t)>v(r, t)$. This means that the initial assumption of the black hole hypotheses about the penetration of the falling particle inside $r_{g}$, based on the statement that the $\tau$-evolution can proceed independent on the $t$-evolution, is incompatible with GR $[9,10]$ since in GR both times are related across the constraint $r(\tau) \equiv r(t)$ leading to the dependence $\tau(r(t)) \equiv \tau(t)$ and then to $\tau(t)<\tau_{g}$.

\subsection{Freezing of a dust shell with formation of a simplest frozar}

In the previous section the asymptotic approach of a test particle to $r_{g}$ was studied for the case of falling outside the star $r(t)>r_{b}(t)$, in the region where the field in $K_{0}$ is static, which allowed us to use the exact solution of the problem. Turning to the star's evolution, let us study the questions about evolution of the worldline of a particle on the star's surface $r(t)=r_{b}(t)$, and about perspectives crossing $r_{g}$ by the star's surface.

In GR, as well as in the Newtonian theory, the problem of a particle on the star's surface has an exact solution due to four properties of gravitation: a) the field outside the spherical star is independent on a radial distribution of its matter, b) on the surface the internal solution for the field is matched with the external static solution, c) the contraction speed is maximal at the free fall of particles, and d) the field inside a spherical shell is constant.

These circumstances allow us, on the one side, to use a dust matter approach, since the presence of pressure only slows down the contraction. On the other side, they allow us to begin with a model of a thin spherical shell, where: a) the internal solution is trivial (constant), b) the field on the surface is given by the external solution, c) dust 
particles at the same time are sources of the field and test particles, so here the knowledge of the test particle's trajectory allows to define structure of the source too. So, the general properties of compact relativistic objects in GR can be revealed on the exactly solved model of the thin dust sphere falling in the self gravitational field [9].

Outside and on the dust shell the metrics is given by the Schwarzschild solution. Inside the shell the metrics is flat, i.e. spatial contractions are absent, while the proper times delay factor is constant everywhere and is equal to its value on the surface. Since the motion of a particle on the surface coincides with the motion of a particle in the static field of the shell, everything that was obtained above for such particle is valid for the dust shell as whole too. Let us briefly describe them by applying to this case.

At falling of the dust shell from relatively large distance $r_{b}\left(t_{0}\right) \gg r_{g}$, at $t_{0}=0$ and $\tau_{0}=0$, the worldline of radially falling particle on the shell $r_{b}=r_{b}(\tau) \equiv r_{b}(t)$ in terms of two times $\tau$ and $t$ (at $t \gg r_{g}$ ) is expressed in the form Eqs. (2)-(3). Both formulas describe the same particle in the same point $\left(r_{b}, \theta, \varphi\right)$ in terms of two times and, therefore, by excluding from them $r_{b}$ we obtain for $\tau(t)$ a relation exactly as in (4).

At any $t<\infty$ from (4) we have $\tau(t)<\tau_{g}$, where $\tau_{g}$ from (5) formally corresponds to the moment $t=\infty$, i.e. the moments of $t$ in the infinite interval $t \in[0, \infty)$ one to one correspond to the moments of $\tau(t)$ in the finite interval $\tau(t) \in\left[0, \tau_{g}\right.$ ) (Fig. 1). Because of invariance of the worldlines of particles the dust shell never crosses $r_{g}$ for any observer with any coordinate corresponding to $t<\infty$ and, thus, in GR the events for the contracting shell with $\tau(t)>\tau_{g}$ do not exist.

In the internal area of the dust shell $r<r_{b}(t)$, where the metrics remains flat during all the time of contraction and the time delay factor is equal to the value on the surface, the event horizon also does not appear, but all test particles will be frozen in those points where they have appeared up to a shell's freezing time near $r_{g}$. Therefore, for the test particles (of non-zero mass) in the shell's gravitational field the spacetime interval remains timelike $d s^{2}>0$ at any spatial point $r \geq 0$.

Let's consider the trajectories of the particles of the contracted shell on the plane $(t, r)$ showed in the Fig. 2. In the previous section it was a particle's worldline, and now it is position of the dust shell at every moment $r_{b}=r_{b}(\tau) \equiv r_{b}(t)$, but all analysis remains fair for the shell too. The freezing of the shell is appeared as an apparent "termination" of the worldline $r(\tau)$ near $r_{g}$, while in fact the evolution is continued in the form of asymptotic approaching $r_{b}(\tau(t))=r_{b}(t)>r_{g}$. Thus, the contraction of the dust shell leads to formation of simplest frozar - a gravitationally-frozen shell with the flat internal metrics where the test particles in the shell are frozen also in those positions where they have placed before freezing.

Such conclusions, analogous to the dust shell, hold in the cases of a thin shell of weakly charged particles or a rotating (neutral or charged) shell falling in the self field. There are two forms of the equation of trajectory in terms of the world time or the proper times of the falling particles. These equations of the trajectory again will give the equations relating the proper times $\tau(t)$ with the world time moments $t<\infty$, the particles on the surface also remain outside an area where the proper times practically 
freeze. In the case of the charged shell - outside an effective gravitational radius $\tilde{r}_{g}(r)=r_{g}-G e^{2} / r$, and in the case of the neutral rotating shell - outside the ergosphere, and the proper times freeze with respect to $t$.

\subsection{Freezing of a dust ball with transition to the frozar state}

At transition to stellar models it is necessary to use solutions of the field equations in a star at a given equation of state. Since practically all realistic solutions are nonstatic, there is a question whether it is possible to use the static frame $K_{0}$ with its world time $t$.

The answer occurs positive because of two circumstances. First, gravitation of a spherical star does not change length of standard rods along 2-spheres and they remain the same as for the distant observer and, therefore, in such star we can continue to use the radial coordinate $r$ determined through the length of circumference. Secondly, solutions of the field equations allow one to relate the proper time moments $\tau(r)$ of internal layers $r<r_{b}$ with the proper time moment at the surface $\tau\left(r_{b}(t)\right)$ and through it with $t$. This allows one to define both internal and external events $(r(t) \geq 0)$ on the hypersurface of simultaneity $t=$ const..

In the model of a contracting dust ball the standard solution has been found by assigning for each layer $R$ of two functions $f(R)$ and $F(R)$ proportional, accordingly, to initial energy of the layer and mass of this layer (see [3], notations from [6]):

$$
\left(\frac{d r}{d \tau}\right)^{2}=f(R)+\frac{F(R)}{r} \text {. }
$$

Let's consider a case with $f(R)<0$ (elliptic trajectories) for a uniform dust ball, particles of which had rested $\dot{r}\left(R, t_{0}\right)=0$ at the initial moment $t_{0}=0$ (see [6], Problem to $\S 103)$. Let's show in this case transition to the frozar state in the form of a gravitationally-frozen uniform ball. The solution is given parametrically by the equation for a cycloid. Let at the initial moment $\tau_{0}(R)=0$ cycloid's parameter is $\eta_{0}=0$, and the circumference of the surface is equal to $2 \pi r_{0}$. The choice of the functions $f(R)$ and $F(R)$ in the form:

$$
f=-\sin ^{2} R, \quad F=2 a_{0} \sin ^{3} R,
$$

leads to the isotropic Friedmann's solution with a scale factor $a(\eta)$, where parameter $\eta(t)$ is defined as $d \tau=a(\eta) d \eta$, and the linear element takes the form:

$$
d s^{2}=a(\eta)\left[d \eta^{2}-d R^{2}-\sin ^{2} R \cdot\left(d \theta^{2}+\sin ^{2} \theta d \varphi^{2}\right)\right] .
$$

An initial value $a(0)$ is defined from matching with the external metrics of the ball which gives $a_{0}=r_{0} / 2 \sin R_{0}$, and the ball's gravitational radius is $r_{g}=r_{0} \sin ^{2} R_{0}$, where $R_{0}$ is the parameter for the surface. Here the isotropy and homogeneity of the ball reveal in the fact that values of $\tau(t)$ at the moment $t$ do not depend on $R$ and are the same for all layers:

$$
\tau(t)=\frac{r_{0}^{3 / 2}}{2 r_{g}^{1 / 2}}[\eta(t)+\sin \eta(t)], \quad r(t, R)=\frac{r_{0}^{3 / 2}}{2 r_{g}^{1 / 2}}[1+\cos \eta(t)] \sin R .
$$


This means that the ball represents a spherically cut part of the uniform Friedmann world in the case of the closed model in its contraction phase. Matching with the external metrics allows one to define the dependence $\eta(t)$ too, but in the form $t(\eta)$ :

$$
\frac{t}{r_{g}}=\ln \frac{\left(r_{0} / r_{g}-1\right)^{1 / 2}+\operatorname{tg}(\eta / 2)}{\left(r_{0} / r_{g}-1\right)^{1 / 2}-\operatorname{tg}(\eta / 2)}+\left(r_{0} / r_{g}-1\right)^{1 / 2}\left[\eta+\frac{r_{0}}{2 r_{g}}(\eta+\sin \eta)\right] .
$$

Now it is essential to what a limit tends the proper time $\tau(t)$ at $t \rightarrow \infty$. As shows (12), at $t \rightarrow \infty$ we have $\tan (\eta / 2) \rightarrow\left(r_{0} / r_{g}-1\right)^{1 / 2}$ and $\eta$ tends to a finite limit:

$$
\left.\eta(t)\right|_{t \rightarrow \infty} \rightarrow \eta_{g}=2 \arccos \left[\left(r_{g} / r_{0}\right)^{1 / 2}\right] .
$$

This means that the moments in the infinite interval $t \in[0, \infty)$ one to one correspond to the values of the cycloid parameter in the finite interval $\eta(t) \in\left[0, \eta_{g}\right)$. As the ball contracts and its surface closely approaches $r_{g}$, there occurs the exponentially fast freezing of the surface under the law (11). The internal layers freeze under the same law, since, due to homogeneity and isotropy, the proper times everywhere are the same. Then for the value $\tau_{g}$, at which the proper times freeze asymptotically, we find:

$$
\begin{gathered}
\left.\tau(t)\right|_{t \rightarrow \infty} \rightarrow \frac{r_{0}^{3 / 2}}{2 r_{g}^{1 / 2}}\left(\eta_{g}+\sin \eta_{g}\right) \equiv \tau_{g}, \\
\tau_{g}=\frac{r_{0}^{3 / 2}}{r_{g}^{1 / 2}} \arccos \left[\left(r_{g} / r_{0}\right)^{1 / 2}\right]+r_{0} \cdot\left(1-r_{g} / r_{0}\right)^{1 / 2} .
\end{gathered}
$$

Internal layers of the ball freeze at the values of radial coordinate:

$$
r(\infty, R)=\frac{r_{0}^{3 / 2}}{2 r_{g}^{1 / 2}}\left(1+\cos \eta_{g}\right) \sin R=\left(r_{0} r_{g}\right)^{1 / 2} \sin R .
$$

As we see, the surface of the ball freezes beyond $r_{g}$, i.e. $r\left(\infty, R_{0}\right) \rightarrow r_{g}$, while internal layers occur to be distributed sinusoidally at different distances from the ball's center. Further world lines of ball's particles will remain almost parallel to the axis $t$ and to each other (Fig. 2). To obtain this diagram, from (11) we find $\eta(r)$

$$
\eta(r)=\arccos \left(\frac{2 r}{r_{0} \beta(R)}-1\right),
$$

where $\beta(R)=\sin (R) / \sin \left(R_{0}\right)$, and by substituting it into (12), find $t(r)$.

For the scale factor $a(t)$ and its limiting value $a_{g}$ at $t \rightarrow \infty$, when the ball freeze, remaining as uniform, we obtain:

$$
a(t)=[1+\cos \eta(t)] r_{0}^{3 / 2} / 2 r_{g}^{1 / 2},
$$

with $a_{g}=\left(r_{0} r_{g}\right)^{1 / 2}$. A relation $a(t) / a_{g}$ changes in the interval $\left[0, \eta_{g}\right)$ as:

$$
\tilde{a}(t) \equiv \frac{a(t)}{a_{g}}=\frac{r_{0}}{2 r_{g}}[1+\cos \eta(t)] .
$$

The limiting density we find from initial density: $\rho_{g}=\rho_{0} a_{0}^{3} / a_{g}^{3}=\rho_{0} r_{0}^{3} / r_{g}^{3}$. 
Thus, the homogeneous dust ball with the standard idealizations and the initial conditions, leading to the Friedmann metrics, freezes very quickly near the proper time moment $\tau(t) \rightarrow \tau_{g}$, the radial coordinates of the layers $r(t, R) \rightarrow\left(r_{0} r_{g}\right)^{1 / 2} \sin R$ and the density $\rho(t) \rightarrow \rho_{0} r_{0}^{3} / r_{g}^{3}, \quad$ so that at $t \rightarrow \infty$ already there never happens with this ball. Thus, the dust ball passes into the frozar state - a star with gravitationally frozen internal structure which remains almost unchanged at its coexistence with other objects of the Universe at any $t<\infty$.

So, the uniform dust ball with the standard idealization and entry conditions conducting to the Friedmann metrics frozen very quickly at values of proper times $\tau(t) \rightarrow \tau_{g}$, radial coordinates of layers $\quad r(t, R) \rightarrow\left(r_{0} r_{g}\right)^{1 / 2} \sin R \quad$ and density $\rho(t) \rightarrow \rho_{0} r_{0}^{3} / r_{g}^{3}$, so at $t \rightarrow \infty$ already more never happens nothing to this ball. Thus, the dust ball passes into the state of a frozar - a star with completely frozen internal structure which is almost not changing at its coexistence with other objects of the Universe at any time $t<\infty$.

For the dust ball the above considered isotropic Friedmann solution is applicable only approximately since in fact at the central symmetry the acceleration, directed to the center, is non-zero and isotropy is broken by it, the time component of the metrics depends on $r$ and proper times are slowed down non-uniformly.

These properties are taken into account in more consecutive solution of the Einstein equations given by Oppenheimer and Snyder (OS) [4], which will be considered below. At $t \gg r_{g}$ a relation of two times $\tau(t)$ in this solution in the reversed form is:

$$
t \approx-r_{g} \ln \left[\frac{1}{2}\left(\frac{R^{2}}{R_{b}^{2}}-3\right)+\frac{R_{b}}{r_{g}}\left(1-\tau / \alpha_{b}\right)^{2 / 3}\right]+O\left(r_{g} / t\right),
$$

where $R_{b}$ is the value of this variable on ball's surface, $R=0$ at the center, and $\alpha_{b}=2 R_{b}^{3 / 2} / 3 r_{g}^{1 / 2}$. Values of $R$ for other layers we find from:

$$
r(t)=R \cdot\left[1-\tau(t) / \alpha_{b}\right]^{2 / 3} .
$$




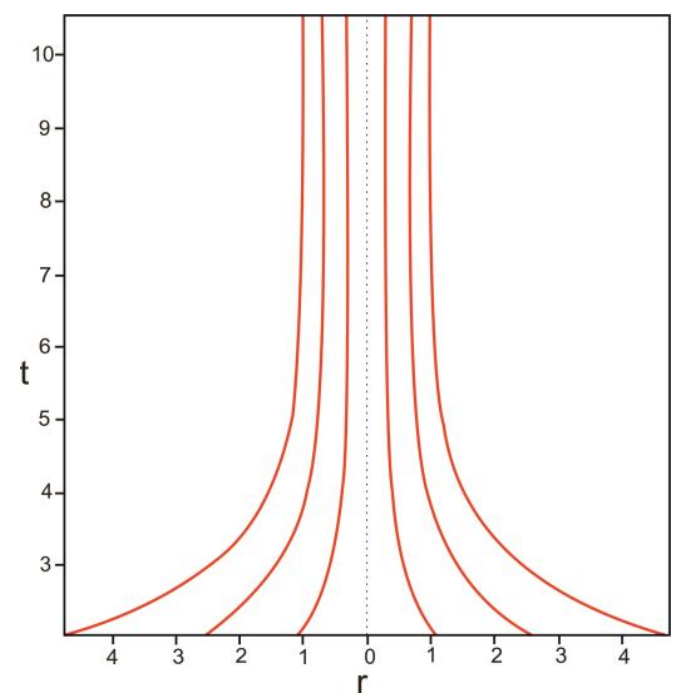

Fig. 3. Freezing of a dust ball in the OS solution in terms of the worldlines of particles (in units $r_{g}$ ). The surface approaches $r_{g}$, but does not cross it, other layers freeze at different distances from the center. After freezing the worldlines of all particles evolve parallel to $t$-axis and among themselves.

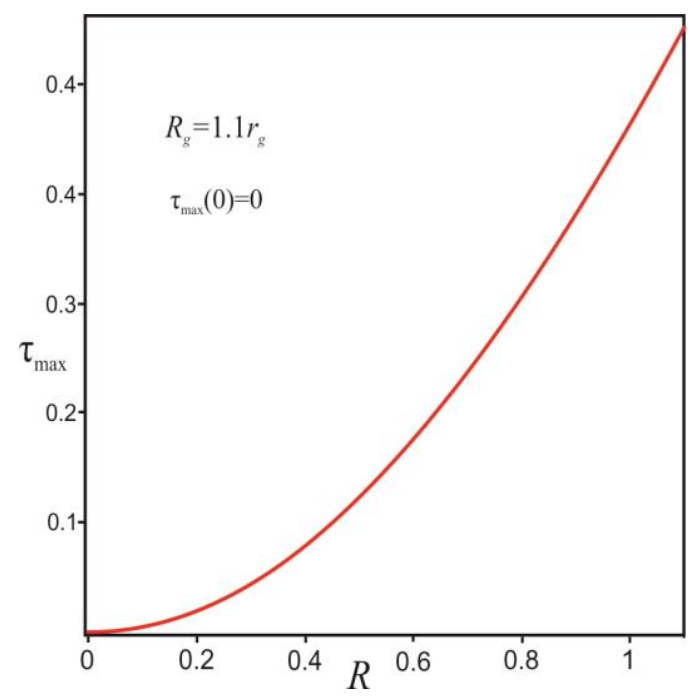

Fig. 4. Non-uniform freezing of proper times of dust ball's layers with respect to the freezing moment $\tau_{\max }(0)$ of the center.

For obtaining a diagram for worldlines $t(r)$ we find from (21) a dependence $\tau(r)$ :

$$
\tau(r)=\alpha_{b}\left(1-r^{3 / 2} / R^{3 / 2}\right)
$$

and put into (20), which gives:

$$
t \approx-r_{g} \ln \left[\frac{1}{2}\left(\beta_{R}^{2}-3\right)+\frac{r}{\beta_{R} r_{g}}\right],
$$

where $\beta(R)=R / R_{b}$. These worldlines are shown in Fig. 3 .

For understanding of a physical meaning of the dependence (20) it is more useful a direct form $\tau(t, R)$, where the proper time of each layer is expressed through $t$ :

$$
\tau(t, R)=\alpha_{b}\left\{1-\frac{r_{g}^{3 / 2}}{R_{b}^{3 / 2}}\left[4 e^{-t / r_{g}}+\frac{1}{2}\left(3-\frac{R^{2}}{R_{b}^{2}}\right)\right]^{3 / 2}\right\} .
$$

At $t \gg r_{g}$ the proper times of layers exponentially quickly freeze near maximal values:

$$
\tau_{\max }=\alpha_{b}\left\{1-\left[\frac{r_{g}}{2 R_{b}}\left(3-\frac{R^{2}}{R_{b}^{2}}\right)\right]^{3 / 2}\right\} .
$$

The diagram of the dependence $\tau_{\max }(R)$ is shown in Fig. 4. As we see, the proper time at the surface $R=R_{b}$ freezes at the value:

$$
\tau\left(t, R_{b}\right) \rightarrow \alpha_{b}\left(1-r_{g}^{3 / 2} / R_{b}^{3 / 2}\right),
$$

whereas at the center of the ball $(R=0)$, where the proper time delay is stronger, it asymptotically freezes earlier the surface at a value $\tau_{\max }(0)<\tau_{\max }\left(R_{b}\right)$ :

$$
\tau(t, 0) \rightarrow \alpha_{b}\left[1-\left(3 R_{b} / 2 r_{g}\right)^{3 / 2}\right],
$$


quickly (in terms $t$ ) approaching to the limit:

$$
\Delta \tau_{\infty}=\tau\left(\infty, R_{b}\right)-\tau(\infty, 0)=(\sqrt{3 / 2}-2 / 3) r_{g} \approx 0,558 r_{g} .
$$

Thus, at any distribution of density in the ball the time delay factor is maximal at the center and minimal on the surface and the center freezes faster than the surface.

The radial contraction factor in the OS solution is given by:

$$
e^{-\lambda(r, t)}=1-\frac{R^{2}}{R_{b}^{2}}\left[4 e^{-t(r) / r_{g}}+\frac{1}{2}\left(3-\frac{R^{2}}{R_{b}^{2}}\right)\right]^{-1}
$$

At the center $R=0$ we have $\lambda(0, t)=0$ at any moment. In the higher layers the radial contraction factor grows and as the surface closely approaches to $r_{g}$, the physical volume quickly increases because of the radial contraction in the higher layers. Thus, the radial contraction factor in the higher layers increases with $t$ exponentially $e^{\lambda(r, t)} \rightarrow e^{t(r) / r_{g}}$. Separate layers of the dust ball, with different $R$ at the beginning, quickly freeze (i.e. do not depend on $t$ ) at the values of the radial coordinate:

$$
r=\frac{R}{R_{b}}\left[\frac{r_{g}}{2 R_{b}^{1 / 3}}\left(3-\frac{R^{2}}{R_{b}^{2}}\right)\right]^{3 / 2} .
$$

Thus, in process of ball's contraction together with increase of physical volume and number of particles in the higher layers, the proper time delay effect in the lower layers will increases. The freezing of all processes, having begun at the center, will extend on the higher layers also, so the contracted dust ball in terms of $t$ quickly freezes at the surface radius very close to $r_{g}$ but outside it $r_{b}>r_{g}$, and further its structure on entire volume remains unchanged, i.e. OS solution also leads to the frozar formation. Trajectories of particles are timelike $d s^{2}>0$ in ball's volume, the surface lies always outside $r_{g}$ and, therefore, particles and radiation in principle can leave the dust ball.

\subsection{Freezing of a star with nonzero pressure by transition to frozar}

The frozar formation process in collapse at zero pressure (a thin dust sphere and a dust ball), considered above in two sections, has a general character and at a soft equation of state the conclusions for collapse at non-zero pressure will be mainly the same. Below we show that for two standard exactly solvable stellar models including the pressure.

Let us consider a static homogeneous star of mass close to a critical mass, when at adiabatically increasing of mass $r_{g}$ grows and approaches the surface radius $r_{b}$. Really, $r_{g}$ grows with mass $M$ linearly $r_{g}=2 G M$, while $r_{b}$ grows slowly $r_{b} \sim M^{1 / 3}$, thus $r_{g}$ approaches $r_{b}$. As a rule, there is a critical value of $r_{b}$ (for example, $r_{c}=9 r_{g} / 8$ ) at which stability could be lost, but it is also rather close to $r_{g}$.

Let matter density in a cold uniform star is constant: $\rho(r)=\rho_{0}=$ const. In this case time component of the metrics $g_{00}(r)$ and pressure $p(r)$ are expressed through $r$ and mass $M=4 \pi \rho_{0} / 3 r_{b}^{3}$ in the standard form [7]:

$$
g_{00}(r)=\frac{1}{4}\left[3\left(1-r_{g} / r_{b}\right)^{1 / 2}-\left(1-r_{g} r^{2} / r_{b}^{3}\right)^{1 / 2}\right]^{2},
$$




$$
p(r)=\frac{3 M}{8 \pi r_{b}^{3} g_{00}^{1 / 2}(r)} \cdot\left[\left(1-r_{g} r^{2} / r_{b}^{3}\right)^{1 / 2}-\left(1-r_{g} / r_{b}\right)^{1 / 2}\right] .
$$

The time component of the metrics $g_{00}(r)$ decreases from the surface to the center and, vice versa, the proper time delay factor, which is equal to $g_{00}^{-1 / 2}(r)$, is minimal on the surface and maximal at the center:

$$
\begin{gathered}
g_{00}^{-1 / 2}\left(r_{b}\right)=2\left[3\left(1-r_{g} / r_{b}\right)^{1 / 2}-\left(1-r_{g} / r_{b}\right)^{1 / 2}\right]^{-1}, \\
g_{00}^{-1 / 2}(0)=2\left[3\left(1-r_{g} / r_{b}\right)^{1 / 2}-1\right]^{-1}>\left(1-r_{g} / r_{b}\right)^{-1 / 2}=g_{00}^{-1 / 2}\left(r_{b}\right) .
\end{gathered}
$$

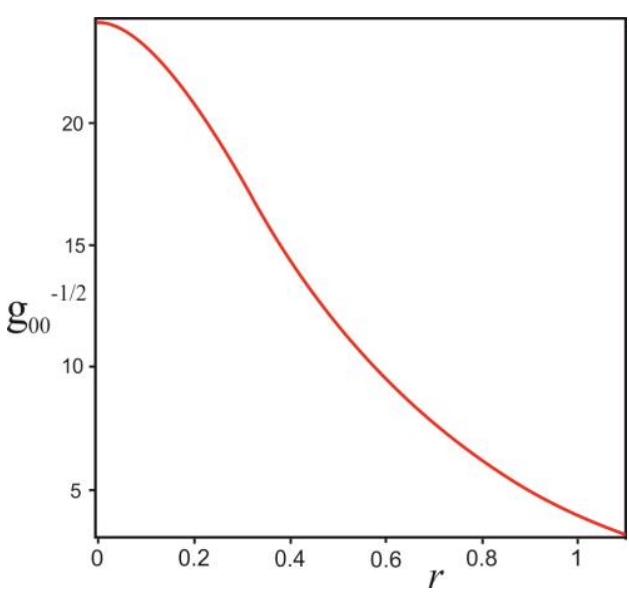

Fig. 5. Heterogeneity of the proper time delay factor in a star with incompressible liquid $\left(r_{b}=1.1 r_{g}\right)$.

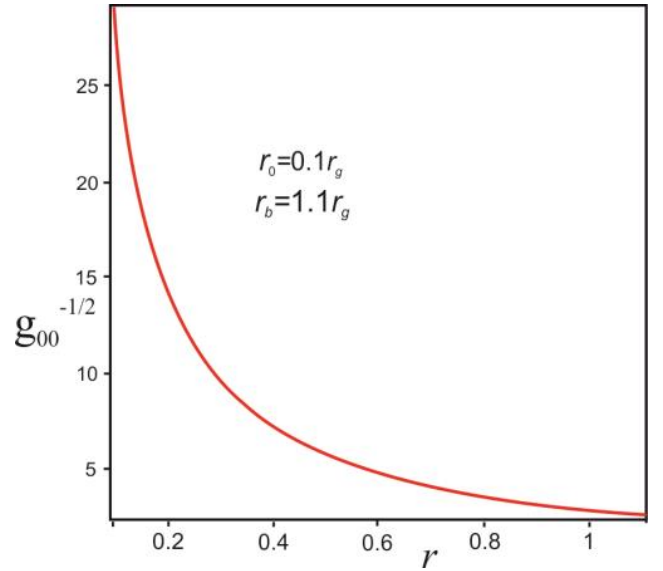

Fig. 6. Heterogeneity of the proper time delay factor in a star with ultrarelativistic matter.

The diagram of growing of $g_{00}^{-1 / 2}(r)$ to the center is presented in Fig. 5. As we see, even if the star loses stability and its surface becomes very close to $r_{g}$, where the proper time begins to freeze exponentially quickly, the proper times of particles near the center and in other internal layers freeze before and more quickly than at the surface. Thus, while we neglect a possible scenarios of the stabilization or anticollapse and consider a worst ("pessimistic") scenario, when contraction is inevitable, the including of pressure reveals only on a dependence of the time dilation factor on the layers, but it does not change the character of the star's freezing as a whole with formation of the frozar.

Let's consider another model of a relativistic star, the main part of matter in which is the ultrarelativistic particles with the equation of state $p(r)=\rho(r) / 3$. In such star the temperature effects are essential everywhere due to the equation of state $\rho(r)=\sigma T^{4}(r)$, however, for estimations we will continue to consider star's dynamics as for a cold case, by returning to the temperature effects in the next part of the paper.

The time component of metrics on the star's internal layers has a simple form [4]:

$$
g_{00}(r)=\frac{r}{r_{b}}\left(1-\frac{2 G M\left(r_{b}\right)}{r_{b}}\right)=\frac{4}{7} \frac{r}{r_{b}}, \quad r \leq r_{b} .
$$

On the surface the proper time delay factor is small, while at the center vicinity increases as $1 / r^{1 / 2}$ and even diverges (Fig. 6):

$$
g_{00}^{-1 / 2}\left(r_{b}\right)=\sqrt{7} / 2, \quad g_{00}^{-1 / 2}(0) \rightarrow \infty
$$


At the central regions of such star the proper times of particles already are frozen even long before the star's contraction. In fact, the center of the star with ultra-relativistic matter always is in the frozar state (at neglecting by the temperature effects). At contraction of the star with quick freezing, this already existing internal frozar near the central region will extend by including higher layers as surface approaches close to $r_{g}$, after which the star already completely turns into the frozar state.

\section{Relativistic heating at contraction of stars and relativistic explosion}

\subsection{Heating at contraction and relativistic explosion of supermassive stars}

In the first part of the paper it was considered contraction of a cold matter (dust or with pressure) where the temperature effects have been neglected that has led to full and eternal gravitational freezing of a star with transition to the frozar state. Turning to more consecutive accounting of the temperature effects, further we will see that they can become a crucial factor, and in GR the star's contraction may be quite hot.

The metrics of any spherical non-rotating (and electrically neutral) star on its surface is smoothly matched by the Schwarzschild metrics and, therefore, even at the accounting of temperature and pressure, if they are insufficient for stopping of the contraction, the surface will continue to approach $r_{g}$ asymptotically, never reaching it. In addition to this general property, it follows from simplest idealized models of collapse in GR that at enough strong contraction there appears a new general properties, not depending on the equations of state $[9,10]$ :

1) the time dilation factor on the surface is much more less than at the center:

$$
e^{-v(0) / 2} \gg e^{-v_{s}\left(r_{b}\right) / 2}=\left(1-r_{g} / r_{b}\right)^{-1 / 2},
$$

2) the radial contraction factor on the surface is much more than at the center (where there is no contraction):

$$
e^{\lambda\left(r_{b}\right) / 2} \gg e^{\lambda(0) / 2}=1
$$

To define, how these general properties will change the equations of state and dynamics of a star, at first we will consider a case of a supermassive star. In this case, as a surface approaches $r_{g}$, a mean density still remains small and locally the ideal gas law remains valid. In a layer between $r-\Delta r$ and $r$ the density is related with the layer's local volume as $\rho=\Delta M / \Delta V$. At first, we will consider a case when mass $\Delta M$ does not change during contraction. Then we can include this mass and all factors into $b=$ const. and the equation of state for this layer takes the form:

$$
p(r)=b \cdot T(r) / \Delta V,
$$

where $p, T$ are pressure and temperature.

As surface closely approaches $r_{g}$ the local temperature near the surface growths exponentially with $t$ :

$$
\frac{T\left(r_{b}(t)\right)}{T\left(r_{b}(0)\right)}=\frac{e^{v_{b}(0) / 2}}{e^{v_{b}(t) / 2}} \sim e^{t / 2 r_{g}} .
$$

At falling from $r_{b}(0)$ up to $r_{b}(t)$ in a local static frame, the energy of the layer near surface will be subjected to the gravitational violetshift. If in the case of a dust the gravitational energy led only to increasing of the falling layer's kinetic energy, in gas a part of this kinetic energy transforms into heat and the layer's temperature increases. 
But, because of radial contractions now in a layer from $r^{\prime}-\Delta r$ up to $r^{\prime}$ there will place in $e^{\lambda(r) / 2} / e^{\lambda\left(r^{\prime}\right) / 2}$ times more local standard radial "rods" and thus the local volume element $\Delta V^{\prime}$ will increase:

$$
\frac{\Delta V\left(r^{\prime}\right)}{\Delta V(r)}=\frac{e^{\lambda\left(r^{\prime}\right) / 2}}{e^{\lambda(r) / 2}} \cdot \frac{r^{\prime 2}}{r^{2}},
$$

As a result, the particles of the gas with higher local mean energy will be distributed in a larger physical volume and, consequently, it follows from (39)-(41) that the local pressure near the surface does not depend on the relativistic factor:

$$
p_{b}\left(r^{\prime}\right)=b \frac{T_{b}\left(r^{\prime}\right)}{\Delta V_{b}\left(r^{\prime}\right)} \simeq \frac{r^{2}}{r^{\prime 2}} p_{b}(r),
$$

Let's consider what happens near the center of such star. As a surface approaches $r_{g}$, a local temperature near the center increases anyway faster than on the surface. But now there are practically no radial contractions and in a layer $\Delta r$ near the center a local volume element practically does not contain a relativistic factor and is independent on $z$ :

$$
\frac{\Delta V_{c}\left(r^{\prime}\right)}{\Delta V_{c}(r)} \simeq \frac{r^{\prime 2}}{r^{2}}
$$

As a result, particles of gas with higher mean local energy will be distributed in the same local volume and, consequently, according to (39), (40) and (43) at contraction the star's local pressure near the center contains the relativistic growing factor the same as for temperature:

$$
p_{c}\left(r^{\prime}\right)=b \frac{T_{c}\left(r^{\prime}\right)}{\Delta V_{c}\left(r^{\prime}\right)} \simeq\left(1+z^{\prime}\right) \frac{r^{2}}{r^{\prime 2}} p_{c}(r)
$$

Thus, in process of contracting of a supermassive star of small density is happens exponentially rapid (in terms $t$ ) increasing of the local temperatures inside the star, sufficiently larger than on the surface $T(r) \geq T_{b} \sim z \sim e^{t / r_{g}}$, which leads to the nonuniform increasing of the local pressures. The local pressure near the surface changes insufficiently, whereas the local pressure at the center increases exponentially rapidly $p_{c} \sim z \sim e^{t / r_{g}}$, which at some stage leads to stopping and expansion of the star's internal layers close to the surface with their further expansion.

Let's note a new and nontrivial relativistic effect which consists in that at the first stage there is an increasing of concentration of main part of star's mass-energy near its surface by formation of almost empty center and a massive thick shell near the surface.

\subsection{Heating at contraction and relativistic explosion of superdense stars}

For superdense stars a hot phase arises for the same reasons as in a homogeneous cosmological model. As well as in cosmology, in the model of a contracting star at a hot phase to the total energy there contribute mainly the ultrarelativistic particles.

This allows us to accept the equation of state for the ultrarelativistic matter $p=\rho / 3$ as quite realistic for a hot stage of a stellar mass collapsing object practically at entire volume with the exception of a small "core" of radius $\delta r_{c}$ and a thin crust at the surface. Then the time component of metrics in this intermediate area $\delta r_{c}<r<r_{b}-\varepsilon$ takes the form (35). Near the surface the time delay factor $g_{00}^{-1 / 2}\left(r_{b}\right)$ is finite, while near 
the core it is very large $g_{00}^{-1 / 2}\left(\delta r_{c}\right) \sim g_{00}^{-1 / 2}\left(r_{b}\right) / \delta r_{c}^{1 / 2}$. Therefore, if at the first approximation we consider the contraction as adiabatic with reaching a thermodynamic equilibrium, a local temperature $T(t)$ will be distributed in the volume as:

$$
T(t)=g_{00}^{-1 / 2}(t) T_{0}=b \frac{T_{0}}{r^{1 / 2}} .
$$

On the surface it should be the Schwarzschild value of the metric that gives for the redshift's growth $z \sim e^{t / r_{g}}$ and this value is minimal one for inner layers of the star. Growth of the local temperature at internal layers stronger and, consequently, also will be proportional to $z$ on the surface:

$$
T(t)=\frac{T_{0}}{\sqrt{g_{00}(t)}} \sim e^{t / r_{8}} T_{0 b} \sim z T_{0 b} .
$$

At such exponentially rapid growth of $z$ on the surface with the same growth of local temperatures, the matter into star transfers into the hot radiation-dominating phase and the local values of pressure also grow exponentially:

$$
p(t) \sim T^{4}(t) \sim e^{4 t / r_{g}} p_{0} .
$$

Growth of the local temperature opens channels of transformations of particles and also leads to the phase transitions. Energy liberation at these reactions and phase transitions can lead to star's stabilization at some equilibrium value of parameters or to inverse expansion of upper layers or entire star.

At transition to quark phase, firstly of central layers, and then the upper layers with the energy releasing allowing to stop the contraction or leading to large ejections and explosion of the star, the layers with quark matter of $\mathrm{u}, \mathrm{d}$ quarks have approximately on $1 / 3$ higher energy, than the neutron matter. This energy gap allows us to consider a model of light quark stars as a probable for a part of compact objects heavier neutron stars.

In the literature there are many evidences that at numerical simulation, by using the considered and other equations of state, such ball can transfer into the expansion state.

\subsection{Big Bang as relativistic explosion of the Universe}

For possibilities of the hot contraction and the relativistic explosion there is, although heuristic, but strong observational evidence. From the above considered simplified model of a star as cutting from Friedmann's homogeneous world, it follows logically that a hot phase at the contracting will be essential characteristic for the star's evolution too. Thus, from the GR model of the Universe, hot at past, follows the model of the collapsing homogeneous star hot at future.

Thus, if we consider the Big Bang (BB) as a recollapse after a hot phase of the Universe, this means that the same should be supposed for the relativistic star too - the collapse can lead to subsequent recollapse. This means that the star can recede from a hot phase by experiencing a relativistic explosion, which can be called as a Local Bang (LB).

A reality of the optimistic scenario of the relativistic collapse can follow from set of arguments and observational facts, such as:

(1) as an alternative to the optimistic phase of the collapse GR leaves only a frozar state, almost completely losing activity almost forever;

(2) observations confirm activity of compact objects and a possibility of the relativistic explosion or recollapse - except the Big Bang, other classes of powerful local explosions in astrophysics are known with rapid and unusually huge energy release, do not explained by usual or at least known mechanisms; 
(3) the existence of the active phase of the collapse is admitted by the equations of GR, from the standard stellar models and numerical simulations follows that at contracting of enough massive objects the pressure near the center grows more rapidly than at upper layers that can lead to quasi-stability or expansion at first central and then higher layers.

All these testify that in GR, beginning from some critical mass, the relativistic explosion perhapse inevitable, otherwise would not be BB too. Therefore, the problem is not about the existence of the explosion after the hot phase, in principle it is admitted by GR and already observed as BB, but in what of fundamental properties of the relativistic gravitation is responsible for that beginning from some critical mass the collapsing star with the growing at the center pressure to be fated to heating and expanding.

\section{Gravitational crystallization at different scales}

\subsection{Impossibility of merging of frozars and gravitational crystallization}

At radial falling of ordinary matter on a formed frozar will lead to the growing of its mass and the gravitational radius only, while at radial falling of two and more already frozen compact objects toward common center of inertia there appears a new situation. In the Newtonian gravity compact objects can merge by forming more massive compact object. In GR at approaching of two and more frozars (or glowsars) they cannot merge and fully freeze at some distance from each other by forming a frozen cluster. It is clear already on an example of three frozars, where the gravitational radius of the system approximately three times exceeds the surface radius of each of frozars (Fig 7).

Particles of the Planck energy also turn to a frozon state - the particles, fluctuations of which are frozen in their self gravitational field $[9,10]$ and also cannot merge, by forming frozen microclusters.

Let's consider two identical frozars of mass $m$ and surface radius $r_{b}$, which are very close to their gravitational radii $r_{b}>r_{g}=2 G m$, and let they are freely falling to a common center of inertia which almost twice less than that gravitational radius of the two-frozar system.

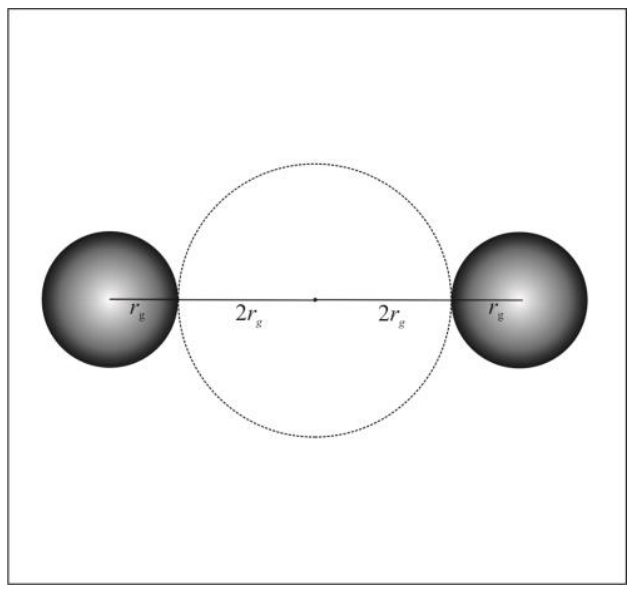

Fig. 7a. Gravitational freezing of two frozars of equal mass on the gravitational radius of the system $2 r_{g}$.

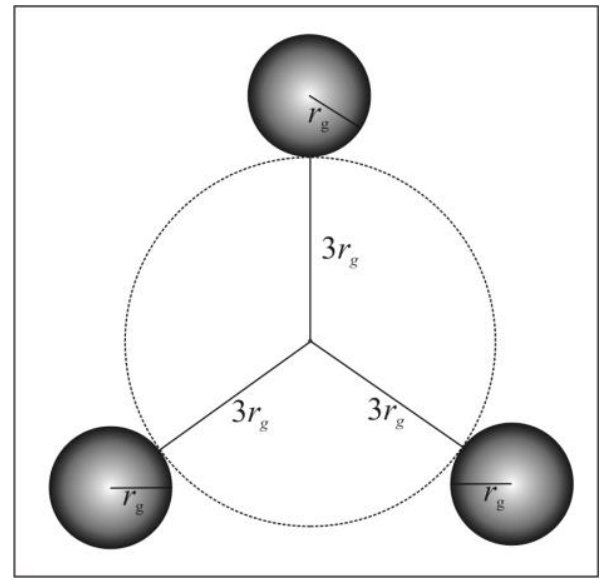

Fig. 7b. Gravitational freezing of three frozars of equal mass on the gravitational radius of the center of inertia $3 r_{g}$.

For simplicity, while we suppose that initially two frozars were rested not so far (few $r_{g}$ ) and that their masses are defined without gravitational mass defect. The 
accounting of this mass defect due to the presence of the neighbor will lead to the decreasing both $m$ and $M$, thus without loss of generality we may take:

$$
M \approx 2 m, \quad r_{b}>r_{g} \approx \bar{r}_{g} / 2
$$

In the process of their approaching a distance between the center of inertia of the system and closest points of surfaces of the frozars will remain always larger than the gravitational radius of the system $\bar{r}_{g} \simeq 2 r_{g}$ because of stronger freezing of the proper times. As a result, at freezing of the system as a whole there will remain some distance between the nearest points of surfaces of the frozars of order $4 r_{g}$ and, consequently, even two frozars cannot merge, so at sufficient closing they will quickly form a frozen twofrozar cluster.

In cases of three and four (symmetrically placed) frozars, as triangle or tetrahedron, the system's gravitational radius will exceed the gravitational radius of each of frozars three or four times and there one cannot talk about closing enough - the system freezes in a state when distances between neighbors are equal several radiuses of each of frozars.

In cases of three or four frozars, placed symmetrically as a triangle or a tetrahedron, the gravitational radius of system will be several times exceeds the gravitational radius of each frozar and they also can not to approach - the system freezes at the state when distance between neighbors averagely several times larger than the radius each of frozars.

Interesting configurations can form a two frozar system with another frozar or a frozar cluster where two frozars have been joined and frozen before approaching of other neighbors. As a result, at approaching two frozar system the remaining single frozar or the cluster freeze on rather large distance from it.

Thus, at approaching of frozars there will be formed frozen two- or many-frozar clusters - gravitational crystals - where separate frozars or two frozar clusters freeze at some distance, having be slightly flattened, and world lines of their centers and all remaining points will evolve be almost mutually parallel. Therefore, in astrophysics and cosmology, at modeling of the structure of the most compact gravitationally-frozen massive or supermassive clusters of frozars and ordinary matter, in addition to ordinary processes, it should be necessarily taken into account the formation of the frozen gravitational crystals too.

When falling other mass of the smaller size on a gravitational crystal from two and more frozar, this masses is frozen in limited volume and not dissolved in a crystal. Thereby process of growth of a gravitational crystal when falling new portions of matter happens by local freezing of this mass that leads to already new stage of crystallization when separate sites were not frozars earlier. Such hybrid of frozar with a ordinary matter can expand to very big sizes and masses, forming supermassive frozars in the form of a gravitational crystal.

\subsection{Gravitational crystallization at the Planck distances}

The gravitational radius of elementary particles $r_{g}(E)=2 G E / c^{4}$ linearly grows with their energy $E$, whereas their quantum mechanical wavelength $\lambda_{q}(E)=h c / E E$ is inversely proportional to $E$. At the value of energy $E_{g}=E_{p l} / 2$, where $E_{p l}=\left(h c^{5} / G\right)^{1 / 2}$ - the Planck energy, "gravitational diameter" of a particle $2 r_{g}\left(E_{g}\right)$ becomes equal to particle's wavelength $\lambda_{q}\left(E_{g}\right)=h c / E_{g}$ : 


$$
\lambda_{q}\left(E_{g}\right)=\frac{h c}{E_{g}}=\frac{4 G E_{g}}{c^{4}}=2 r_{g}\left(E_{g}\right) .
$$

Here $r_{g}\left(E_{g}\right)$ is equal to the Planck length $r_{g}\left(E_{g}\right)=l_{p l}=\left(G h / c^{3}\right)^{1 / 2}$.

In the region of space $2 r_{g}\left(E_{g}\right)$, where energy of a particle of an order $E_{g}$, there is a gravitational freezing of quantum fluctuations, and high-frequency fluctuations of energy exceeding $E_{g}$ occur to be excluded due to strong gravitational redshift. In quantum field theory this leads to the effective cutting of integrals on energy and momentum of virtual quanta in loop diagrams $[9,10]$. Thereby gravitation leads to the universal and invariant regularization of all fields and naturally excludes divergences in the loop diagrams of quantum field theory. This fact explains high efficiency of the perturbation theory in quantum electrodynamics in which, at such gravitational cutting, one-loop contributions give few percent from the "tree" diagrams contribution.

Particles of energy of order the Planck energy $E_{g}$, frozen in the self strong gravitational field, further we will call as frozons. Notice that at emission of quanta with smaller energy frozon will be "refrozen" since its rest energy will decrease also and the latter becomes as for ordinary particles, practically without gravitational freezing effects. Let's consider further only a model case when it is supposed the existence of particles of energy close to $E_{g}$ and where the effects of freezing dominate, slowing down the decay processes too. Transition of the virtual particles with energy $E_{g}$, created in the loop diagrams of quantum field theory, to the frozon state is fundamental restriction of standard GR for particle physics, not depending on further possible graviton contributions.

There is a question: can two, three and more frozons merge by forming particles of higher energy? Formally, the problem is reduced to the existence in quantum field theory, accounting the general-relativistic freezing effects, some "quasilocal" (in the sense of localization in the Planck volume) interaction vertexes for frozons. The answer appears unexpected - such vertexes practically do not exist and frozons cannot merge, moreover, the existing frozon practically cannot decay on two or more frozons. It is again is related by the freezing of a two-frozon system at approaching by each of frozons the gravitational radius of the system. Such closing up will lead only to formation of two- or many-frozon frozen atoms in which all processes are strongly slowed and practically frozen. These frozon atoms, at joining, can form frozon microcrystals.

Observable consequences of such gravitational crystallization may be various, but simplest consequence is that frozons and frozon "atoms", if they exist, can be appeared as a "dark matter". With ordinary matter and radiation they will interact mainly pure gravitationally and practically will not participate in other interactions. It is related by, at first, compensating by vacuum polarization effects almost all their quantum numbers, thus the many-frozon structures will be formed mainly across the condensation of particle-antiparticle pairs, and secondly, difference between their neighbor energy levels are of order of the Planck energy, and thirdly, if something will be happen, it will last in world time much more longer than other ordinary processes.

Thus, the gravitational crystallization without merging is the basic process which will dominate in the systems of several approaching frozons and the formed frozon microcrystals, together with single frozons, can be a part of "the dark matter". 


\subsection{Cosmological consequences of gravitational crystallization}

Primordial frozons and their microcrystals, formed at early periods after the Big Bang, practically will not be "refrozen" during expansion and further they will reveal as a dark matter. This dark matter formation mechanism, which will appear in many structures, does not need in introduction of new types of fields and particles since it is enough to consider transition to the frozon state of the known quanta in extreme conditions at beginning of the cosmological expansion.

In the closed models of the Universe in the contraction era there will dominate supergalaxies with bright nucleus and large number of compact objects, especially, frozars and frozar supercrystals. Since most of matter will concentrate in compact objects, such as white dwarfs and neutron stars, merging of them form at first stage single frozars, and after closing of latters there will form many-frozar supercrystals. Similar processes with nuclei of galaxies and quasars will lead to the formation of the semi-frozen supermassive gravitational crystals.

Thus, the contraction era will differ sufficiently from the expansion era by that main part of matter will freeze in the form of frozar supercrystals with more and more growing effective gravitational radii. Eventually these supercrystals will prevent the further contraction of the Universe as a whole and contracting to a very small volume will not happen. At formation of frozar supercrystals and their merging finally into a total supercrystal in scales of the Universe, the Great Collapse stops with transition into the Great Freezing.

However, if before that some processes will lead to stopping of contraction and to further expansion, a new cycle will begin. But, this cycle will not be similar any more to previous one since the most of matter will remain in the form of frozar supercrystals which do not depend in any way on the cosmological expansion and this frozen matter will reveal as supermassive nuclei of galaxies and quasars.

One of commonly accepted beliefs assigned to the standard GR is the statement about formation of initial or final cosmological singularity in the contracted state. Usually it is believed that the problem arises when process is described by comoving observers in terms of their proper times. But in this case also the consecutive application of GR allows one to remove this problem.

In fact an origin of the problem is that till now it is supposed that the Universe was expanded from and can contract up to the Planck volume $V_{p l}$. However each of separate "point" particles is an "external observer" with respect to other particles and at approaching together up to $l_{p l}$ their proper times appear as slowed down with respect to world time of a center of inertia of the given many particle system.

Therefore, in fact in GR it is necessary to take into account, that each of $N_{0}$ present particles and additionally creating $\Delta N$ quanta contract up to the proper Planck volume $V_{p l}$, and after that wavelength of these particles never decreases. As a result, at the contraction of the Universe, smallest really achievable volume $V_{\min }$ is sum of these elementary volumes $V_{p l}$ of each of particles and in units of the Planck volume it always will exceed a product of $V_{p l}$ and the present number of particles in the Universe:

$$
V_{\min }>N_{0} V_{p l} \sim 10^{88} V_{p l} \text {. }
$$


At reaching of this minimal volume $V_{\text {min }}$ the particles will be frozen because of self gravitational fields and the cosmological contraction will stop in terms of cosmological proper time too.

In cosmology there is also the problem of large value of entropy since it has been supposed that the Universe expands from and may contracts up to $V_{p l}$. But if one takes into account that in the closed Universe contraction is prolonged only up to $V_{\min }$ from (50)with number of particles not less the present one, in GR the problem with large value of entropy disappears. Large number of elementary volumes at the smallest contracting and corresponding "number of sells" just characterize entropy of the Universe which is further conserved too, as well as there conserves the residual mean total number of particles in later periods of expansion.

\section{Astrophysics of frozars and relativistic explosion}

\subsection{The expected and observed properties of stellar mass frozars}

In fields of ordinary stars and white dwarfs gravitational time delay is insufficient. Only for neutron stars this phenomenon becomes remarkable, but here also it can be taken into account only as a correction.

However, at masses exceeding $3 M_{\odot}$ and density of an order of the neutron star density, the star's surface is so close to $r_{g}$ that the gravitational freezing becomes dominating. Such object, whether it will be a hot neutron star or a quark star, if during contraction keeps sufficient mass, further almost completely freezes and becomes a frozar.

As it was shown in the previous sections, at contraction close to $r_{g}$ the surface freezes outside $r_{g}$, asymptotically approaching it, in the metrics on the surface and inside the frozar does not contain the horizon or singularity. All layers occur to be frozen in those positions at which were just before the freezing of the surface, there to be frozen all fields too.

Let's present some of main properties of frozars following from GR for such asymptotically contracting states at their gravitational freezing:

1. The large time delay near the surface of the frozars leads to the absence of the sharp changes of parameters and to a very smooth and stretched in time character of all peaks of intensity of radiation at accretion. Such slowing down can be used for the measuring of time delay factor near the frozars;

2. Silence at a final stage of falling of a matter on frozars because of gravitational freezing as the falling matter, and radiation from it.

3. The probability of freezing without essential loss of mass during contraction grows with growth of mass of a compact object.

Within the last decades searches of compact relativistic objects in the Universe led to detection of tens remnants of stars as candidates for such objects with a masses in the interval $(4 \div 20) M_{\odot}$ that slightly exceeds the limit $3 M_{\odot}$ for stability of cold neutron stars. Estimations give us from $10^{3}$ up to $10^{4}$ of such objects almost in each galaxy [12].

The found supercompact objects of stellar masses are more "silent" than neutron (or quark) stars and the thermonuclear flashes from the falling of matter on their surface do not observed. Some compact supermassive objects, such as galaxy nuclei also are 
"silent". These facts are in agreement with the frozar picture of relativistic stars where the "flashes" and other locally-fast phenomena become strongly slowed down in terms of $t$.

Single frozars radiate mainly at falling on them of matter, but in a general case so weak that it is difficult to find them at direct observations. It is known that a more favorable situation represents a compact object in a binary system with the ordinary star acting as a donor of accreting matter. Because of tidal influence of the frozar the matter of the neighbor flows to it and the frozar in such accretion mode appears as a powerful source of X-ray radiation.

Large number of close binary systems, sources of X-ray radiation, have been discovered, but only about few tens of them have the mass of a compact source exceeding $3 M_{\odot}$ and have an order $(4 \div 20) M_{\odot}$, so they can be to candidates for frozars. Observations showed that the size of these objects does not exceed their several gravitational radii. Observers discriminate them from ordinary neutron stars by lack of attributes of pulsars, and also that they are not X-ray barsters (Type I).

For candidates to frozars the lack of ordinary indications of neutron star's surface is revealed in difference of a form and time variability of their X-ray radiation spectrum. This observational fact is in agreement with the frozar picture of GR and is interpreted not as lack of the surface, but as the gravitational freezing of the falling matter, including the emitting radiation, near already frozen surface of the frozar or the semi-frozen surface of the glowsar.

A gap in mass spectrum of neutron stars and stellar mass frozars at $(2 \div 4) M_{\odot}$, found in observations, also is expected in the frozar picture [12]. It can be estimated from dependence on the mass of the maximal mean density $\rho_{g}=(4 \pi / 3) M / r_{g}^{3} \sim M^{-2}$ to which tends the frozar's mean density at freezing in the area of radius about $r_{g}$.

Firstly, then mass is less, then higher density will be reached before freezing of the star, therefore, temperature and pressure also will be maximal. As a result, in the states of insufficiently strong gravitational freezing, which takes place in the interval of masses $(2 \div 4) M_{\odot}$, there is more rapid growth of temperature effects. This with higher probability leads to destabilization of the contracting star with subsequent ejection of a part of rest energy, and the remained mass will not provide freezing (see section 4.3).

Secondly, since beginning of some value of stellar mass $M_{\mathrm{o}}$, as show observations, approximately $\sim 4 M_{\odot}$, the gravitational radius, linearly growing with mass of stars $r_{g} \sim M$, leads to earlier freezing of the contracting stars. However, mean densities $\rho_{g} \sim M^{-2}$ will be less and less with growth of mass, which can not provide a sufficient warming up of the matter of the stars before freezing, so the frozar formation becomes more and more probable.

Other expected property of the frozar formation is the revealed in observations flatness of mass spectrum of candidates to frozars [12]. It is related by that the probability of freezing at contraction grows with mass, while a number of stars decreases with mass, thus the mass dependence of a product of these probabilities will sufficiently weaker than each of them.

Really, let probability $p_{M}$ that the star has given mass decreases with mass as:

$$
p_{M}(M) \sim 1 / M^{\alpha},
$$


and the probability $p_{f}$ of freezing of the star at contraction, which is non-zero beginning $3 M_{\odot}$, further grows with mass as

$$
p_{f}(M) \sim M^{\beta}, \quad M>3 M_{\odot} .
$$

Then the product of these probabilities gives the probability of formation of frozars of given mass:

$$
p(M)=p_{M} p_{f} \sim M^{\beta-\alpha}, \quad M>3 M_{\odot} .
$$

It is known that the number of stars in the Galaxy decreases with mass very quickly $\left(\alpha_{0} \sim 5\right)$. However, a number those of them which nuclear fuel burned out, for their quantity is less to 1-2 orders than total number of stars and this part grows with mass. Taking this into account, the parameter $\alpha$ for the number of candidates to frozars we can estimate as about: $\alpha \sim 2 \div 3$.

The number of stars, remnants of which have time to freeze gravitationally at contraction, grows with mass also sharply (since $3 M_{\odot}$ ). With growth of mass $\rho_{g}$ decreases, as well as temperature and pressure of all layers, which reduces probabilities of heating up and explosion. The probability of the gravitational freezing with growth of mass only increases. Therefore, it is quite appropriate to put as $\alpha \sim \beta \sim 2 \div 3$ already in the first approximation just beginning $4 M_{\odot}$, which gives:

$$
p(M) \sim \text { const., } \quad M>4 M_{\odot} .
$$

Thus, the simple estimation shows that the mass spectrum of candidates to frozars is flat or close to flat.

Thus, the frozar picture is in agreement with known observational data for compact relativistic objects and also can explain the found new relationships.

\subsection{Supermassive frozars in galaxy nuclei as gravitational crystals}

Density of supermassive compact objects is low and in this sense the situation is simpler than for stellar mass objects. But there are questions also, how correctly applied the relativistic thermodynamics in the strong gravitation states, when heterogeneity of temperature and pressure depend on non-uniform freezing and stretching of layers.

The gravitationally frozen matter will be superdense only in the central parts of supermassive frozars in the centers of quasars, nuclei of galaxies and star clusters. In areas be closer to a surface such density of matter can small, though with the distribution which frozen in terms t. Therefore, supermassive frozars have difficult structure with matter in various phase states.

In observations several hundreds of supermassive objects in nuclei of galaxies with masses $\left(10^{6} \div 10^{10}\right) M_{\odot}$ are found, estimated sizes of which no more than few $r_{g}$. They also are more "silent" than objects having ordinary surface, i.e. they do not reveal flashes from matter falling on the surface.

In the previous part of the paper it was discussed the property of frozars to form clusters without merging. As objects, structure of which is frozen in the self gravitational field, at closing to other frozars they become frozen again, but already in the gravitational field of the system in the sense that now the motion of the center of mass of each of frozars stops at rather large distance between the frozars. As the result, some of supermassive frozars form as such "gravitational crystals" where it is frozen, except initial frozars of stellar mass, large amount of ordinary matter too, in the form of dust, gas and compact stars. 
In observations the effect of growth of estimated mass of supermassive compact objects in the centers of quasars with growth of $z$, i.e. with decreasing of proper age of these objects, was revealed, which at first sight unexplainable from the point of view of continuing accumulation of mass of these objects during accretion of gas, dust and falling of stars [12]. This effect became especially mysterious after discovering more than ten quasars with $z>6$, at which such objects have age less than 1 billion years and for such time could not accumulate so large mass.

This problem is naturally solved in GR at the accounting of a new cosmological effect - the redshift stasis effect in the gravitationally-bound regions, such as galaxy clusters [13]. The matter is that definition of absolute luminosities $L$ of quasars and estimation of their masses from $L$ are model dependent and are based on some assumptions. First one is a choice of the certain cosmological model giving relationship of $z$ and $L$ with distance up to quasars. Second one is an assumption about nature of distribution of radiation and growth of $z$ in the quasi-homogeneous universe, where radiation many times passes galaxy clusters, the large gravitationally-bound regions where the effects of the cosmological expansion practically are absent. In the paper [13] it is shown that the accounting of repeated passing by radiation of the galaxy clusters, where there are no effects of expansion, allows one to explain this effect naturally within the picture of continuous accumulation of mass by supermassive objects.

\subsection{The expected and observed properties of relativistic explosions}

The relativistic explosion is a new phenomenon when ultrarelativistic matter and radiation, partially frozen at contraction in the strong gravitational field of a compact object, after weakening of degree of freezing and transition to ordinary rate of the proper time, comparable with world time, leave object across surface with speed close to the light speed, being only partially slowed down by gravitation.

Before creation of SM of particle physics the compact remains of stars with a mass exceeding $(2 \div 3) M_{\odot}$, a stability limit of cold neutron stars, automatically were considered as the uncontrollably collapsing. This paradigm, which was outdate at once with appearing of CM, by inertia is presenting in the literature on GR and astrophysics, despite large number publications with attempts the modeling of contraction of stars in GR by taking into account temperature effects and quark structure of hadrons.

In fact, GR together with $\mathrm{CM}$ requires consideration at contraction of transition of a cold neutron matter into hot, and further into a quark-hadron and even in purely quark phases. The equation of state and dynamics with such masses, densities and strong fields are studied insufficiently for definite conclusions. Therefore, in theory of compact objects, surfaces of which are close to $r_{g}$, it should be continued searching of mechanisms of stopping of contraction and even recollapse, since in GR they do not forbidden, the processes are highly nonlinear and depending on a combination of several essential factors.

To the expected properties of glowsars and relativistic explosions, following from a warming up and quasi-stability in the semi-frozen states, and also explosion at weakening of freezing, belongs the following:

1. Glowsars have higher local pressures and temperatures, than neutron stars and can radiate "quasi-relic" photons and a neutrino.

2. The heavy elements synthesis can take place in glowsars during long world time by ejection at relativistic explosion, which is the mechanism alternative to the synthesis of elements at supernovae explosion. 
To the astrophysical phenomena which it is difficult to explain with the existing mechanisms, or their mechanisms remain questionable, regard explosions with extraordinary large energy release, such as supernovae explosions of different classes and gamma ray bursts. Whether the part from them belongs to relativistic explosions or they nevertheless can be explained with the ordinary mechanisms, will show further studies. The task of the theory consists in specification of possible scenarios and their observed consequences and still in this list there was no the most interesting scenario predicted by GR - the relativistic explosion of supercompact objects.

Energy losses because of various effects, bursts and explosions at internal phase transitions (also prolonged in $t$ ) can lead to their slow "evaporation" or (at a final stage) to rapid "defrosting" in the form of the relativistic supernovae or hypernovae with high violetshift due to high speed of expansion. Exponential growth of local pressure is characteristic for explosive processes and testifies that when the essential part of energy of contraction is transformed to heat, and then to radiation, a stopping with formation of the glowsar and then expansion with emission of hard radiation (burst) not only become quite probable, but even inevitable.

Thus, if the equation of state leads to the reversal of contraction with transition to expansion, at a final stage, when transition of the proper time rate to the world time rate happens exponentially fast, glowsar passes into the relativistic explosion state, by appearing as relativistic supernova or hypernova. Defrosting of the proper time rate in the object with the radiation dominated matter leads to powerful release of radiation through the surface expanding with a relativistic speed. Some of powerful gamma ray bursts can be a result of such relativistic explosion.

A distinctive feature of such processes is the fact that energy liberation will be comparable with a rest energy of matter and essentially exceeds an energy release at quark-hadron interactions (a nuclear synthesis or quark matter). A radiation flow from the expanding at relativistic velocities plasma (protons, nuclei) becomes hard due to the high violetshift. The strong gravitational redshift of photons reduces the estimations of power of such bursts and grows an estimated distance of up to the source.

The revealed in observations new effects - a flat character of mass spectrum of candidates to frozars (their number should increase at lower masses whereas it does not occur), and also a gap in mass spectrum of neutron stars and stellar mass frozars at $(2 \div 4) M_{\odot}$ - are expected from the point of view of possibility of relativistic explosion of contracting stars. They just also testifies that at insufficiently strong gravitational freezing, which takes place at small masses, more rapid growth of temperature effects can be related just with formation of glowsars and subsequent relativistic explosion.

\section{Conclusion}

Thus, it is reviewed the frozar theory as consecutive general relativistic theory of objects of different scales and masses with extremely strong gravitation. Simple relativistic models of contraction of stars show that than the object's surface is closer to $r_{g}$, than the gravitational freezing of $\tau$ (with respect to $t$ ) is stronger in entire volume. At the soft equation of state, when gravitation dominates, at first freezes the center, then freezing will reach higher layers and after all freezes the surface beyond $r_{g}$ (Fig. 3). Here the proper time moment, when the surface freezes, is slightly less $\tau_{g}$ from Eq. (5). Such object with almost completely frozen structure, where worldlines of particles in all volume are time-like, parallel to the $t$-axis and among themselves, is the frozar. Frozars 
at every moment $t<\infty$ have the surface asymptotically coming nearer to $r_{g}$ at $r_{b}>r_{g}$, a non-uniform structure determined by the equation of state at the moment just before freezing and radial contractions growing to the surface.

Unlike black holes with the horizon and singularity, which appear strictly prohibited by GR, the frozars are observable in principle at any time $t<\infty$ and can emit quanta of very high redshift. Observed types of relativistic astrophysical objects (from stellar masses to nuclei of galaxies, quasars and stellar clusters) can be interpreted in GR as frozars of various internal structure and with rather nontrivial environment.

Unlike the Newtonian picture of merging of compact objects, in GR merging of the gravitationally frozen objects does not occur. The objects, structure of which is frozen in self gravitational field, become frozen secondary in the gravitational field of the system and the motion of center of mass of each frozar stops at sufficient distance from other frozars. Such gravitational crystallization generates a new state of matter at which perhaps are the observed supermassive objects in the centers of galaxies.

In the frozar theory the hard equation of a state at some combination of particles and fields can stop contraction after rather strong freezing (glowsar) or even reversal contraction at some stage with defrosting of proper times that leads to a new class of explosions - to the relativistic explosion.

The frozar theory is in agreement with practically all known observational data on compact relativistic objects and relativistic bursts, and also predicts a number of their nontrivial properties which can be checked at further observations.

\section{References}

1. Schwarzschild K. (1916) Sitz. Preuss. Akad. Wiss., 189.

2. Lemaître (1933) Ann. Soc. Sci. Bruxel., A53, 51.

3. Tolman (1934) Proc. Nat. Acad. Sci. US 20, 169.

4. Oppenheimer J.R., Snyder H. (1939) Phys. Rev. 56, 455.

5. Mizner Ch., Thorn T., Wheeler J. Gravitation. v.1-3, 1973.

6. Landau L.D., Lifshits E.M. Class. Theor. of Fields. 1994.

7. Weinberg S. Gravitation and cosmology. 1972.

8. Frolov V.P., Novikov I.D. (1998) Black Hole Physics. K1.

9. Zakir Z. Teor. Phys., Astrophys. and Cosmol. (2006) 1(3) 45; (2007) 2(1) 1; arXiv:0507.2585.

10. Zakir Z. Teor. Phys., Astrophys. and Cosmol. (2012) 7(1) 1; 7(1) 15; (2013) 8(3) 65.

11. Okun L.B. etc. (1999) Physics-Uspekhi 42(10) 1045; (2000) American J. Phys. 68(2) 115.

12. Cherepashchuk A.M. (2014) Physics-Uspekhi, 57(4) 359.

13. Zakir Z. Teor. Phys., Astrophys. and Cosmol. (2014) 9(3) 106. 